KERNFORSCHUNGSZENTRUM

\author{
KARLSRUHE
}

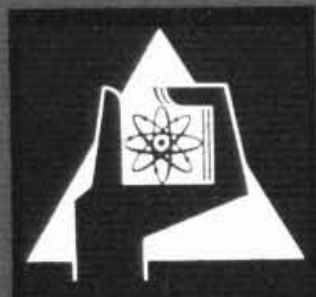




\title{
EIN UNIVERSELLER ZEITGEBER
}

\author{
H. REKER \\ Isotopen-Laboratorium, Kernforschungszentrum Karlsruhe
}

Eingegangen am 15. Februar 1964

A timer is described which gives time-intervals between one and 9999 seconds in steps of one second. The relative accuracy of these intervals is in the range $6 \times 10^{-5}$ and the deviations from an official time-signal with a higher relative accuracy (in the range

\section{Einfuihrung und Aufgabenstellung}

Bei vielen kernphysikalischen Messproblemen spielt die Messung der Zeit beziehungsweise die Herstellung definierter, mit erträglichem Fehler behafteter und gut reproduzierbarer Zeitintervalle eine grosse Rolle. Die Reihe der hierzu gebräuchlichen Zeitgeber reicht von einfachen Stoppuhren bis zu elektronischen Uhren kompliziertester Bauart. Da bei einer grossen Vielzahl von Messaufgaben die Kenntnis des Fehlers der Zeitmessung wichtig ist, erscheint eine gelegentliche Kontrolle der verwendeten Zeitgeber unerlässlich. Auch ist es häufig wünschenswert, dass bei Untersuchungen eines und desselben Problems mit verschiedenartigen Messmethoden die verwendeten Zeitgeber zentral gesteuert werden, so dass der Zeitfehler der einzelnen Messverfahren der gleiche ist. Diese Arbeitsmethode erleichtert u.U. den kritischen Vergleich der erzielten Messergebnisse wesentlich.

Diese Überlegungen führten zum Entwurf und Aufbau eines elektronischen Zeitgebers mit folgenden Eigenschaften und Messmöglichkeiten:

1) Der Zeitgeber wird von einem Quarznormal mit der relativen Frequenzungenauigkeit von $10^{-5}$ gesteuert.

2) Die innerhalb bestimmter Grenzen beliebig wählbaren Zeitintervalle werden mit Hilfe von Untersetzern hergestellt.

3) Das Gerät soll Zeitimpulse zur Steuerung weiterer, vereinfachter Zeitgeber abgeben.

4) Die Genauigkeit des Gerätes soll durch Vergleich mit einem amtlichen Zeitnormal kontrollierbar sein.

5) Die Funktion des Zeitintervall-Untersetzers soll intern kontrollierbar sein.

6) Das Gerät soll die Möglichkeit bieten, normale Feder-Stoppuhren auf ihre Ganggenauigkeit zu kontrollieren.

Die Forderung nach einem möglichst hohen Mass an Zuverlässigkeit und Genauigkeit bei erträglichem Aufwand führte zum Bau eines absoluten Zeitnormals. Die Kontrolle der Ganggenauigkeit des Gerätes erfolgt of $10^{-8}$ ) will be measured. Besides the timer gives time signals with frequencies of $10000,1000,100,10$ of $\mathrm{I} \mathrm{Hz}$ for coupling other time interval-units. A special set gives the possibility for calibrating feather-clocks.

durch Vergleich mit einem um einige Grössenordnungen genauer arbeitenden amtlichen Zeitnormal. Dieses Verfahren bietet den Vorteil, dass durch sinnvolle Vergleichsmessprogramme die Messungsgenauigkeit des vorliegenden Zeitgebers gut bekannt und jederzeit kontrollierbar ist.

\section{Grundsätzlicher Aufbau des universellen Zeitgebers}

Entsprechend den geforderten Eigenschaften besteht der Zeitgeber aus den in Abb. 1 gezeigten Schaltungseinheiten. Das Gerät gliedert sich in die beiden Haupt-Schaltungsgruppen "Zeitgeber" und "Kontrollgerät".

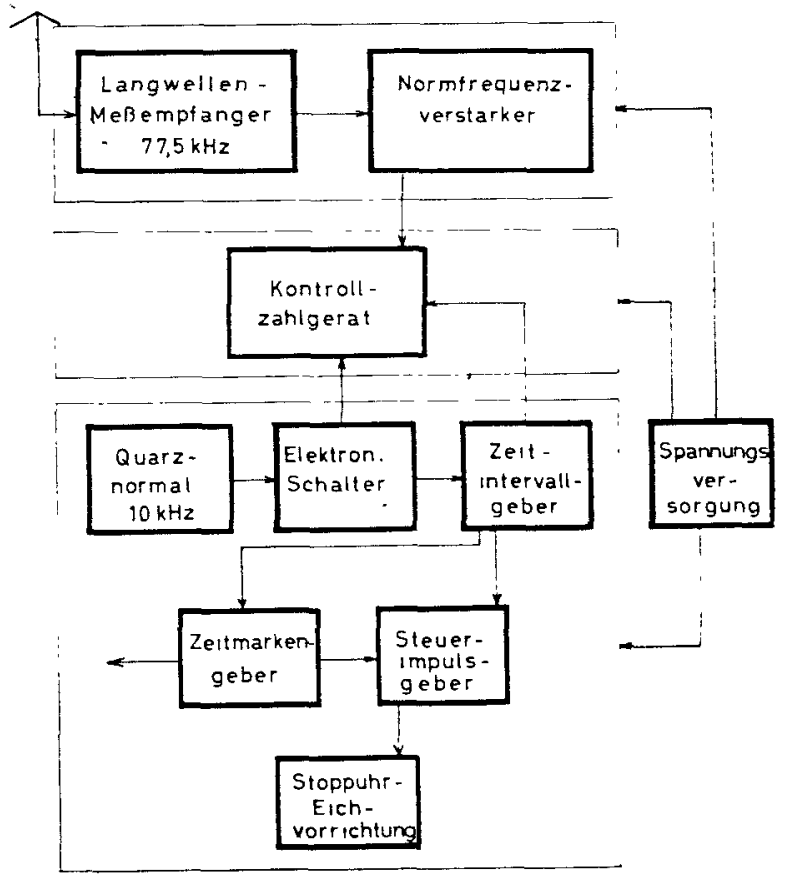

Abb. 1. Blockschaltbild "Universeller Zeitgeber".

\subsection{Grundprinzip des Zeitgebers}

Der Zeitgeber enthält als Mutteroszillator einen 10 $\mathrm{kHz}$-Steuerquarz mit nachfolgendem Verstärker und 
Impulsformer. Eine Koinzidenzstufe dient zur Synchronisierung des den Beginn des wählbaren Zeitintervalls $\Delta T$ bestimmenden Startimpulses mit dem Steuersignal. Ein mehrstufiger Impulsuntersetzer bildet aus dem Steuersignal die gewünschten Zeitintervalle sowie Zeitmarken zur Steuerung beliebig vieler Zeitintervallgeber. Schliesslich steuert der Zeitgeber die Vorrichtung zur Eichung mechanischer Stoppuhren.

\subsection{GRUNDPRINZIP DES KONTROLLGERÄTES}

Das Grundprinzip des Kontrollgerätes und damit auch des Zeitgebers richtet sich zwangsläufig nach der Art des zur Verfügung stehenden Zeitnormals.

\subsubsection{Das amtliche Zeitnormal}

Der von der Deutschen Bundespost betriebene Langwellensender DCF 77 (Trägerfrequenz: $77.5 \mathrm{kHz}$; Leistung: $12 \mathrm{KW}$; Standort: Mainflingen, 50 01'1 Nord, $09^{\circ} 00^{\prime}$ Ost) strahlt werktäglich nach einem bestimmten Programm Messmarken und Normalfrequenzen der Physikalisch-Technischen Bundesanstalt sowie Zeitzeichen des Deutschen Hydrographischen Institutes aus. Aus den innerhalb dieses Programms zur Verfügung stehenden Normsignalen wurde für die Kontrolle des gebauten Zeitgebers das in der Zeit von $11^{10}$ bis $11^{27}$ Uhr ausgestrahlte $200 \mathrm{~Hz}$-Sinussignal ausgewählt. Die von der PTB in regelmässigen Veröffentlichungen nachträglich bekanntgegebene Ungenauigkeit der $200 \mathrm{~Hz}$-Frequenz beträgt relativ im Mittel ca $3 \times 10^{-8}$. Das PTB-Zeitnormal wird von einer Quarz-Uhrengruppe hergestellt, deren Frequenz mit der des Cäsium-Atomresonators (9 $192631770 \mathrm{~Hz}$ ) verglichen wird.

\subsubsection{Prinzip des Kontrollgerätes}

Entsprechend dem vorliegenden Normsignal besteht das Kontrollgerät aus einem Langwellenempfänger zum Empfang des $77.5 \mathrm{kHz}$-Trägers, aus einem 200 $\mathrm{Hz}$-Bandpassverstärker mit Siebgliedern zur Fernhaltung des im Langwellenempfang auftretenden erheblichen Störpegels sowie aus einem Zählgerät zur Registrierung des $200 \mathrm{~Hz}$-Niederfrequenzsignals.

\section{Funktionsbeschreibung des "universellen Zeitgebers"}

\subsection{Der Zeitgeber}

In der Abb. 2 ist das Blockschaltbild des Zeitgebers, gegliedert nach Betriebsfunktionen, dargestellt.

Die von einem quarz-stabilisierten BC-Generator erzeugte $10 \mathrm{kHz}$-Sinusschwingung wird verstärkt und in eine positive Impulsfolge umgewandelt. Der RCGenerator läuft kontinuierlich.

Durch Betätigung der Starttaste $T_{1}$ wird ein Aus-

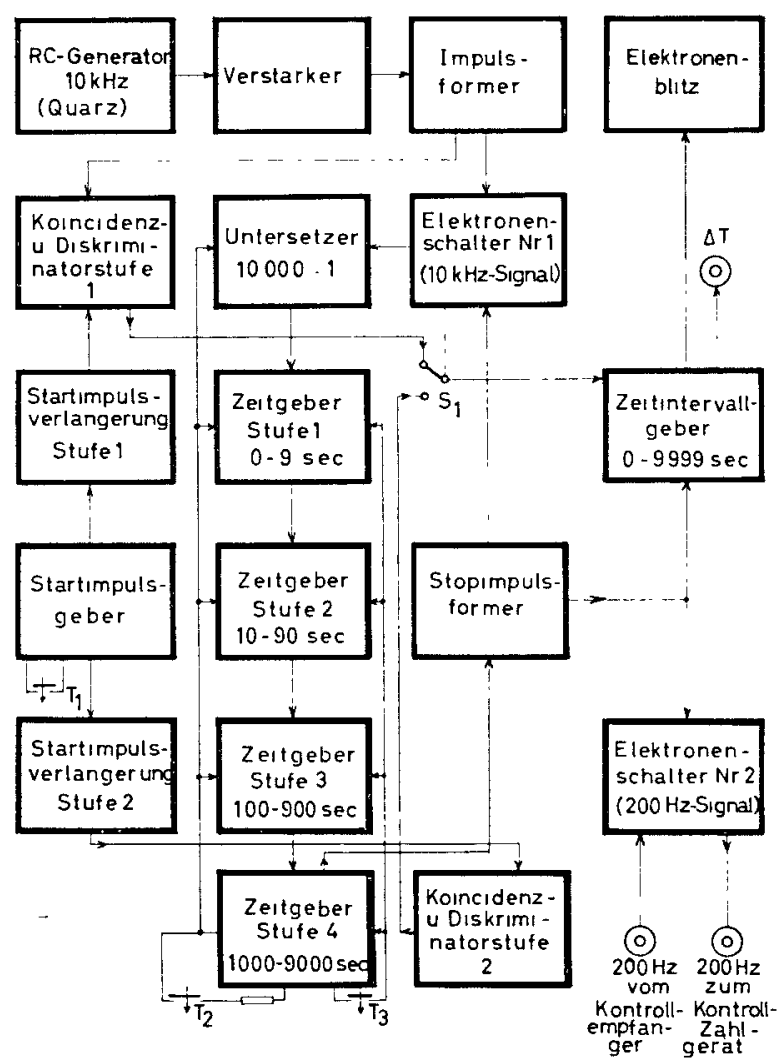

Abb. 2. Blockschaltbild Ze1tgeber.

löse-Impuls erzeugt, damit der elektronische Schalter 1

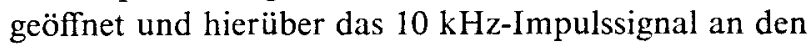
Untersetzer 10000:1 und die nachgeschalteten Zeitgeber-Stufen geführt. Nach Beendigung des am Zeitgeber eingestellten Zeitintervalls wird der elektronische Schalter 1 wieder geschlossen und das $10 \mathrm{kHz}$-Signal dadurch gesperrt.

Der Auslöse-Impuls wird folgendermassen gebildet: Durch Betätigung der Taste $T_{1}$ wird über einen Widerstand ein Kondensator entladen. Der auf diese Weise am Widerstand auftretende Spannungsimpuls wird auf $100 \mu$ s verlängert und mit dem nächstfolgenden Impuls der $10 \mathrm{kHz}-F$ olge in Koinzidenz gebracht. Der die Diskriminatorschwelle $U_{D}$ übersteigende Impuls ist der

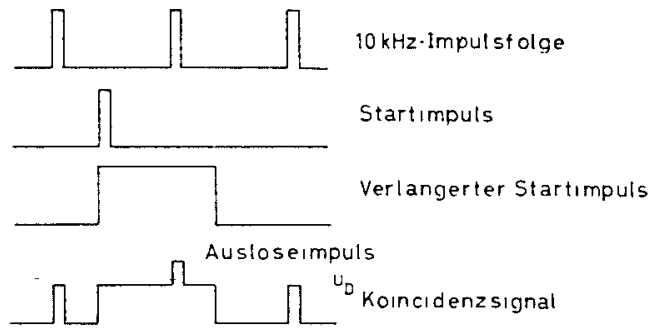

Abb. 3. Bildung des Auslöse-Impulses. 
eigentliche, das Zeitintervall $\Delta T$ beginnende Auslöse-Impuls $s$ (Abb. 3).

Der Zeitgeber selbst besteht aus vier hintereinander geschalteten E1T-Zählstufen, bei welchen die Potentialdifferenz $V_{D^{-}} V_{D^{\prime}}$ mit Hilfe je eines zehnstufigen Spannungsteilers so eingestellt werden kann, dass der Kathodenstrahl eine gewünschte Ziffer trifft (Abb. 4).

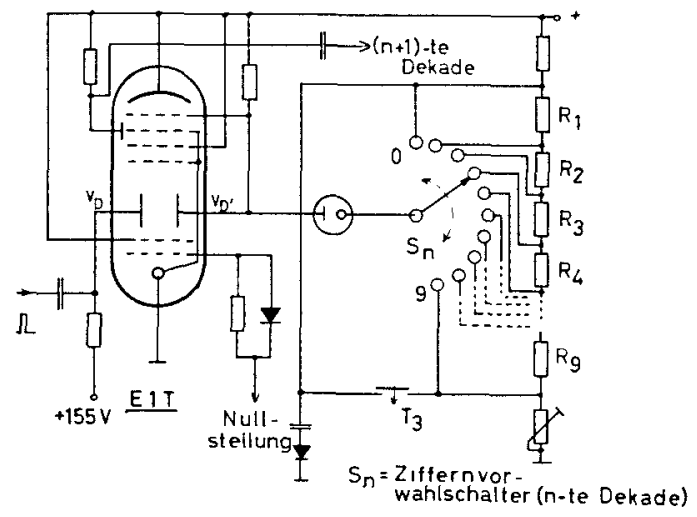

Abb. 4. Ziffernvorwahlschaltung mit E1T.

Diese Ziffernvorwahl wird dann durch Betätigung der Taste $T_{3}$ ausgelöst, nachdem alle ElT-Stufen vorher mit Hilfe der Taste $T_{2}$ auf der Wert "Null" gestellt wurden. Dieses Prinzip ist aus der Zähltechnik als Impuls-Vorwahl bekannt.

Beispielsweise seien die Zeitgeber durch entsprechende Schalterstellung $S$ der vier Dekadenschalter nach Druck auf Taste $T_{3}$ in folgender Weise vorgewählt: Zeitgeberstufe 1: Ziffer 5, Zeitgeberstufe 2: Ziffer 6, Zeitgeberstufe 3: Ziffer 7 und Zeitgeberstufe 4: Ziffer 8. Die Starttaste $T_{1}$ gibt über den elektronischen Schalter 1 eine mittels des 10000:1-Untersetzers hergestellte Impulsfolge der Frequenz $1 \mathrm{~Hz}$ an den Eingang der Zeitgeberstufe 1. Nach 5 Impulsen gibt die E1T der Zeitgeberstufe 1 einen Impuls zur Stufe 2, letztere nach vier von Stufe 1 erhaltenen Impulsen einen Impuls zur Stufe 3, letztere nach drei von Stufe 2 erhaltenen Impulsen einen Impuls zur Stufe 4 und diese schliesslich bildet nach zwei von Stufe 3 erhaltenen Impulsen den Zeitintervall-Endimpuls. Da sämtliche Zeitgeberstufen das Vorwahlpotential nur vor dem Beginn des Zeitintervalls einmalig erhalten, nämlich bei Betätigung der Taste $T_{3}$, arbeiten alle Zählröhren im weiteren Verlauf der ankommenden Impulsfolge nach dem "Aufbrauchen" der vorgewählten Ziffern wie normale Zähler. Mit anderen Worten bestimmt die vorgewählte Ziffer der

Zeitgeberstufe 1 die Anzahl der "Einer",

Zeitgeberstufe 2 die Anzahl der "Zehner".

Zeitgeberstufe 3 die Anzahl der "Hunderter" und Zeitgeberstufe 4 die Anzahl der "Tausender" der an den Eingang der ersten Stufe ankommenden Sekunden-Marken. In dem angeführten Beispiel der Ziffern-Vorwahl 5, 6, 7, 8 ist die Anzahl der notwendigen Impulse zwischen Start und Stop:

$$
\begin{aligned}
& (10-5) \times 10^{\circ}+(10-6) \times 10^{1}+ \\
& +(10-7) \times 10^{2}+(10-8) \times 10^{3}= \\
& =5+40+300+2000=2345
\end{aligned}
$$

entsprechend 2345 Sekunden bei $1 \mathrm{~Hz}$.

Um die Schwierigkeit der Startimpuls-Unterdrückung zu umgehen, wurde die Vorwahlschaltung der Zeitgeberstufe 1 um eine Stelle verschoben, so dass die Einstellung auf den gewünschten Ziffernwert erst durch den Startimpuls erfolgt. Ein Zeitfehler kann dabei nicht entstehen, wenn alle ElT-Röhren des 10000: 1-Untersetzers auf "9" stehen. Der an der Auffangelektrode der letzten Zeitgeberstufe entstehende Impuls ist der Endimpuls des abgelaufenen Zeitintervalls.

Die auf die oben beschriebene Weise gewonnenen Start- und Stopimpulse bestimmen somit das gewünschte Zeitintervall $\Delta T$ und können zur Steuerung beliebiger Messvorrichtungen wie Zählgeräte, ImpulsEinkanal-Spektrometer, Probenwechsler u.ä. verwendet werden.

Zur Prüfung der Zeitintervall-Ungenauigkeit $\delta(\Delta T)$ wird das PTB-Normsignal-200 $\mathrm{Hz}$-während eines beliebigen, beziehungsweise von der geforderten Kontrollgenauigkeit abhängigen Zeitintervalls mit einem normalen Zählgerät registriert. Der Auslöse-Impuls wird jetzt jedoch durch Koinzidenz zwischen Startimpuls und nächstfolgendem Impuls der $200 \mathrm{~Hz}$-Folge gebildet, nachdem der Startimpuls entsprechend verlängert wurde. Dieses geschieht mit Hilfe der Koinzidenz- und Diskriminatorstufe 2. Der verbleibende maximale Zeitfehler beim "Start" von $100 \mu$ s (maximal mögliche Phasendifferenz zwischen dem Kontrollauslöseimpuls und dem nächstfolgenden Impuls der 10 kHz-Impulsfolge) spielt in den Grenzen der durch den Quarz gegebenen Genauigkeit keine Rolle, wenn die Länge des Zeitintervalls für die Kontrollmessung grösser als $10 \mathrm{~s}$ ist.

Wäre z.B. die Länge des Zeitintervalls $\Delta T=100 \mathrm{~s}$, dann registriert das Kontrollzählgerät im Idealfalle 20001 Impulse $(n+1$ Impulse der Periodendauer $\Delta \theta$ Sekunden schliessen einen Zeitraum von $n \cdot \Delta \theta$ Sekunden ein). Da nichts über die Phasenlage des letzten Impulses der $200 \mathrm{~Hz}$-Folge vor dem Stopimpuls bekannt ist, beträgt der maximal mögliche Fehler der Kontrollmessung insgesamt 


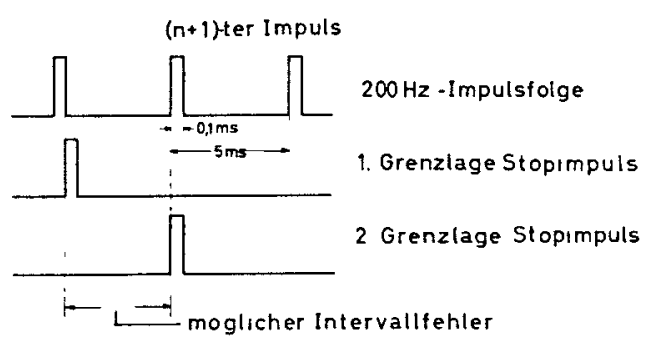

Abb. 5. Systematischer Intervallfehler als Folge der unbekannten Phasenlage des Stopimpulses zum $(n+1)$-ten Signalimpuls.

$$
\delta(\Delta T)=(5-0.1) \mathrm{ms}=4.9 \mathrm{~ms} \quad(\text { Abb. } 5),
$$

d.h. der relative Fehler beträgt bei $\Delta T=100$ Sekunden ca $5 \times 10^{-5}$. Dieser relative systematische Fehler lässt sich durch Verlängerung der Kontrollmesszeit entsprechend verringern. Verwendet man anstelle der 200 Hz-Messmarken den Träger der Frequenz $77.5 \mathrm{kHz}$, welcher mit ebenfalls hoher Genauigkeit $\left(10^{-8}\right)$ bekannt ist, so wird der eben erläuterte Fehler vernachlässigbar klein.

\subsection{DiE STOPPUHR-EICHVORRICHTUNG}

Das Prinzip der Stoppuhr-Eichung mit Hilfe des oben beschriebenen Zeitgebers besteht darin, dass die Uhr im laufenden Zustand zu Beginn und am Ende eines beliebig wählbaren Zeitintervalls $\Delta T$ fotografiert wird. Die Belichtung der Uhr erfolgt mit einem Elektronenblitz, welcher einmal vom Auslöse-Impuls und dann nochmals vom Stopimpuls ausgelöst wird. Dieses Verfahren hat den Vorteil, dass wegen der kurzen

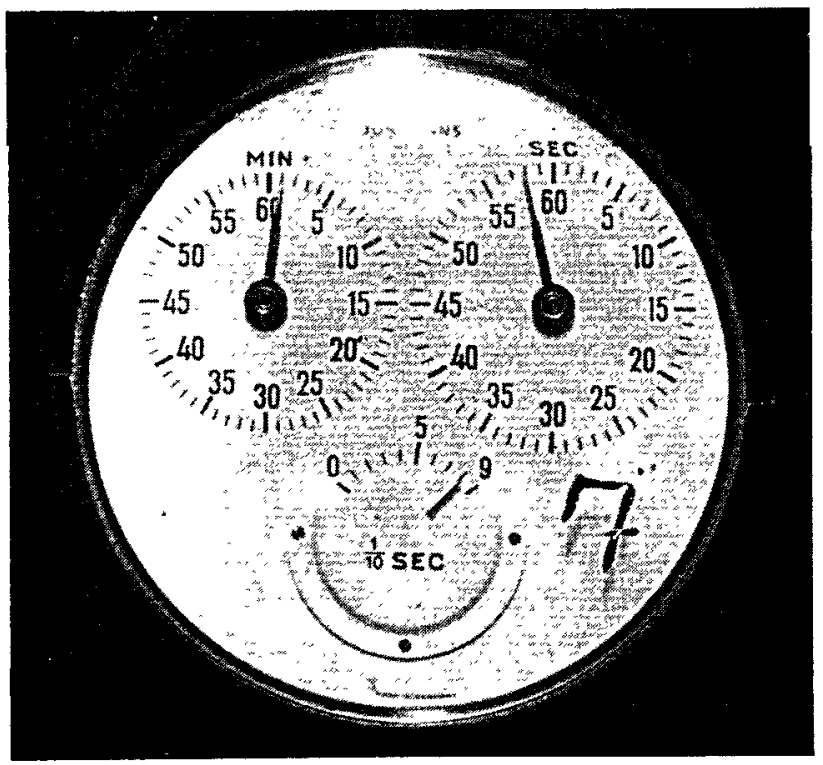

Anstiegzeit und Dauer des Elektronenblitzes (Anstiegszeit ca $10^{-4}$ Sekunden, Dauer ca $10^{-3}$ Sekunden) die Intervallgenauigkeit zwischen den beiden Messmarken erheblich grösser ist, als sie etwa durch mechanische Vorrichtungen zum Starten und Stoppen der Uhr erreichbar wäre. Die Konstruktion der optischen Stoppuhr-Eichvorrichtung zeigt Abb. 6.

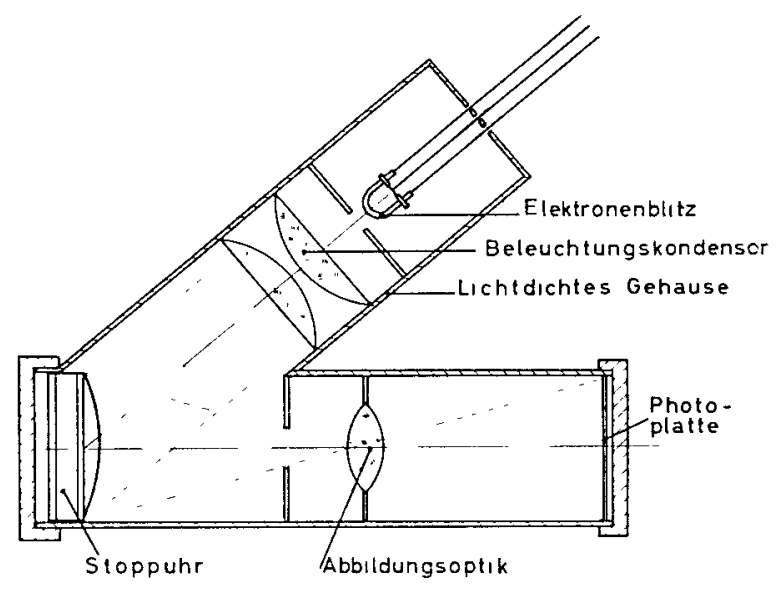

Abb. 6. Stoppuhr-Eichvorrichtung.

Das von der Blitzröhre ausgehende Licht wird durch einen Doppelkondensor in ein paralleles Lichtbündel verwandelt. Der Winkel zwischen Beleuchtungsrohr und Stoppuhrenebene wurde so eingestellt, dass die Reflexionen auf dem Uhrenglas minimal sind. Das hinreichend gleichmässig ausgeleuchtete Stoppuhr-Ziffer-

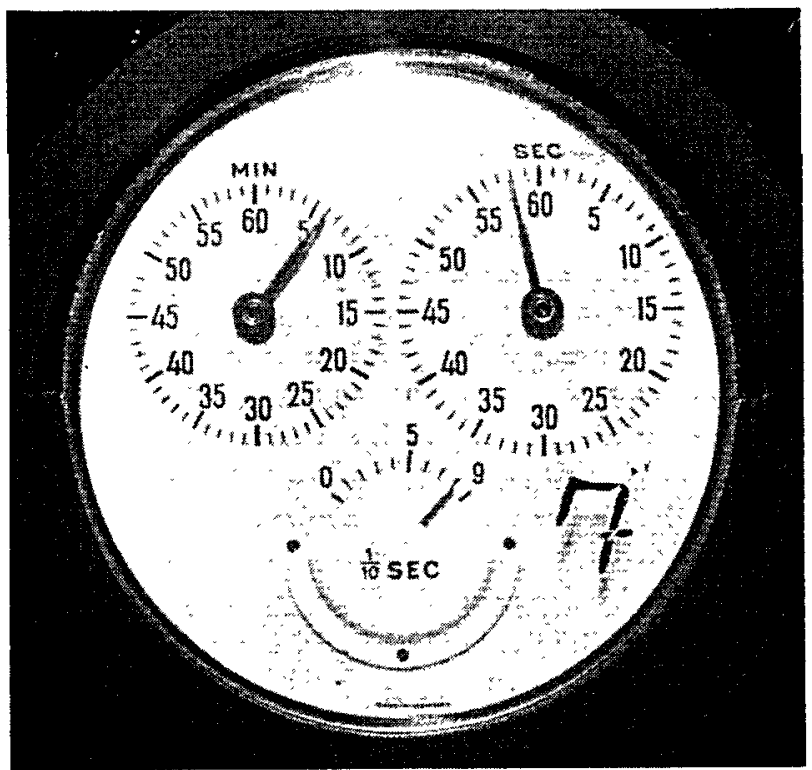

Abb. 7. Stoppuhr-Eichung; Messzeit: $\Delta T=(300.0 \pm 0.02)$ Sekunden. Verfahren mit zwei getrennten fotografischen Aufnahmen. 
blatt wird mittels einer Optik (Brennweite $50 \mathrm{~mm}$ ) auf der Photoplatte abgebildet. Man wechselt entweder zwischen Start- und Stopimpuls die Photoplatte und erhält dann zwei getrennte Aufnahmen (Abb. 7) oder man verschiebt nach der Startaufnahme die Plattenkassette un einige Zentimeter und erhält so Start- und Stopaufnahme auf einer Platte (Abb. 8). Die richtige Belichtung wird mit Hilfe der Blende der Abbildungsoptik eingestellt.

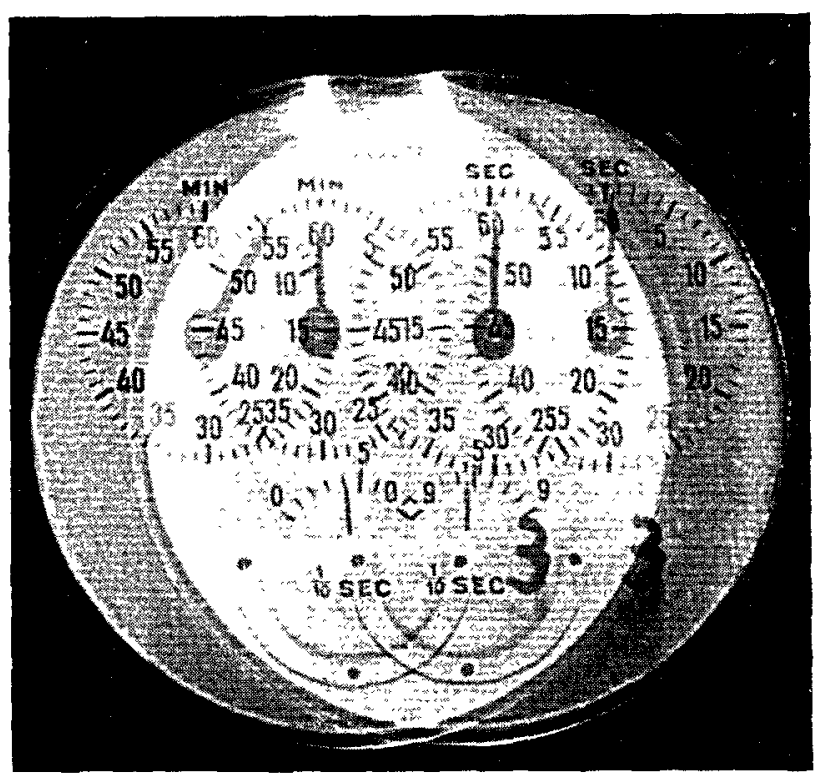

Abb. 8. Stoppuhr-Eichung; Messzeit: $\Delta T=(300.0 \pm 0.02) \mathrm{Se}-$ kunden. Verfahren mit einer fotografischen Aufnahme.

Aus der innerhalb des Eichintervalls auf die oben beschriebene Weise fotografisch festgehaltenen Abweichung des $\frac{1}{10}$ Sekunden-Zeigers lässt sich dann die Ganggenauigkeit der Uhr bestimmen. Stellt man, wie in der Eichung der Abb. 7, keine Abweichung fest ( $\Delta T$ war bei dieser Messung 5 Minuten), so kann man daraus schliessen, dass die Ganggenauigkeit innerhalb der Anzeigegenauigkeit der Uhr liegt. Da die mittlere Ungenauigkeit der vom Zeitgeber gegebenen Zeitintervalle ca $5 \times 10^{-5}$ beträgt, kann erst bei einer Messzeit über 2000 Sekunden (bei Uhren mit einer Anzeigeungenauigkeit von $\frac{1}{10}$ Sekunden) eine Aussage über die Ganggenauigkeit der geeichten Uhr mit einer Fehlerangabe von $5 \times 10^{-5}$ gemacht werden. Nimmt man die Stoppuhreichung während der gleichzeitigen Registrierung des $200 \mathrm{~Hz}$-Normsignals vor, so kann der Messfehler noch kleiner angegeben werden, da dann der absolute Fehler des Eich-Zeitintervalls bekannt ist.

\subsection{DeR MeSSEMPFÄNGER}

Das vom Sender DCF 77 ausgestrahlte Normsignal ist ein mit $200 \mathrm{~Hz}$ amplitudenmoduliertes Langwellensignal der Trägerfrequenz $77.5 \mathrm{kHz}$ entsprechend 3.871 $\mathrm{km}$ Wellenlänge. Für den Empfang dieses Signals wurde ein normaler Langwellenempfänger verwendet, dessen Eingangskreis durch Vergrösserung der Kreisinduktivität der $77.5 \mathrm{kHz}$-Trägerfrequenz angepasst wurde. Das Blockschaltbild des Messempfängers nebst zugehörigem NF-Verstärker zeigt die Abb. 9.

Die Feinabstimmung auf den zu empfangenden Träger erfolgt über einen Drehkondensator mit kleinem Variationsbereich, um leicht eine optimale Abstimmung zu erhalten. Der Empfänger hat insgesamt sechs

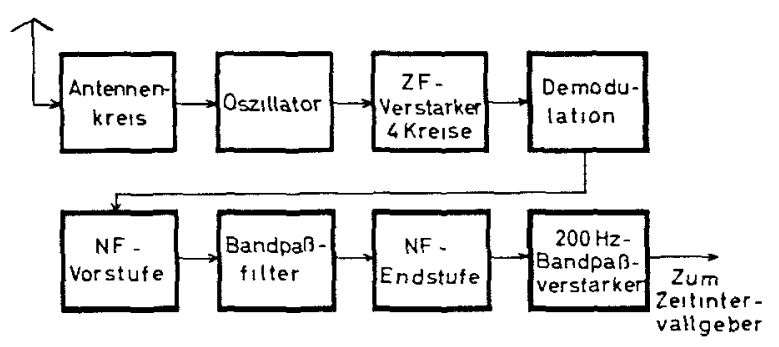

Abb. 9. Blockschaltbild Messempfänger.

Kreise. Der Bandfilter-Zwischenfrequenzverstärker hat vier Kreise mit der Bandmittenfrequenz von $470 \mathrm{kHz}$. Besondere Sorgfalt wurde für die Unterdrückung des im Langwellenbereich sehr grossen Störpegels einerseits und für die Erzielung einer optimalen Trennschärfe andererseits verwendet. Letzteres ist besonders wichtig, da in der Nähe der $77.5 \mathrm{kHz}$-Frequenz das Frequenzband stark besetzt ist.

Die Schaltung zur Unterdrückung von atmosphärischen und Netzstörungen sowie von ausserhalb der 200 $\mathrm{Hz}$ liegenden Modulationen zeigt die Abb. 10.

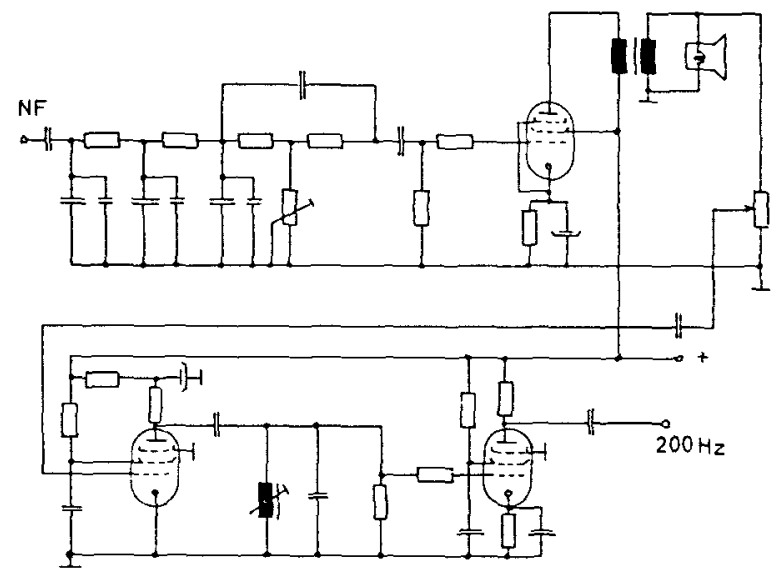

Abb. 10. Resonanz-Bandpassverstärker $200 \mathrm{~Hz}$ mit Störfilter. 
Das von der NF-Vorstufe kommende Niederfrequenzsignal wird über ein Tiefpassfilter auf das Gitter der Endröhre gegeben. Über einen an den Ausgangsübertrager angeschlossenen Lautsprecher kann das Normsignal hörbar gemacht werden. Das an der Sekundärwicklung des Ausgangsübertragers abgegriffene NF-Signal wird dann an das Gitter des aus den beiden Röhren 1 und 2 bestehenden 200-Hz-ResonanzBandpassverstärkers gegeben; dessen $200 \mathrm{~Hz}$-Resonanzkreis wurde so stark gedämpft, dass das durch Störimpulse verursachte Ausschwingen des Kreises um ca $20 \mathrm{~dB}$ unterhalb der Empfindlichkeitsschwelle des Kontrollzählgerätes liegt.

Die Frequenzkurve der Gesamtschaltung Abb. 10 zeigt $\mathrm{Abb} .11$; die gemessene Störimpulsunterdrückung beträgt $25 \mathrm{~dB}$.

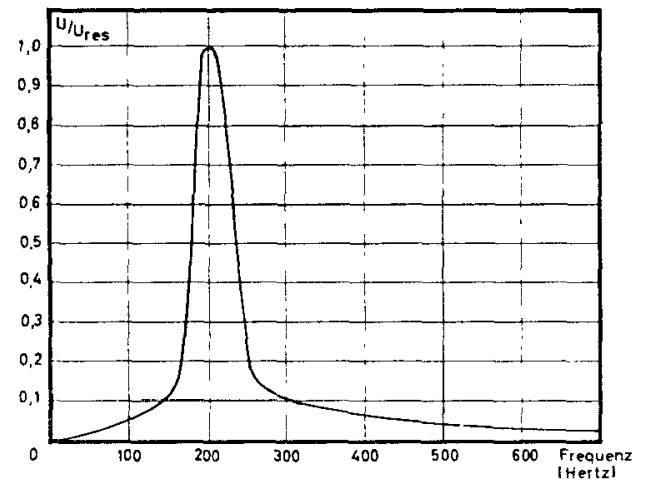

Abb. 11. Durchlasskurve des $200 \mathrm{~Hz}$ Bandpassverstärkers.

Da die Gefahr einer Empfangsstörung über das Netz besonders gross ist, kann der Empfänger im Bedarfsfalle über eine $6 \mathrm{~V}$-Akkumulatorenbatterie gespeist werden.

\subsection{DAS Kontroll-ZäHLGERÄT}

Die Schaltung des Kontroll-Zählgerätes ist mit sechs E1T-Dekaden bestückt nebst einem dreistelligen elektromagnetischen Zähler. Die Impulskapazität dieses Gerätes beträgt somit $10^{9}$ Impulse. Das Zählgerät dient einerseits-wie oben beschrieben-zur Registrierung des PTB-Normsignals. Andererseits ist eine Kontrolle der Schaltfunktion der beiden Elektronenschalter im Zeitgeber möglich, indem das $10 \mathrm{kHz}$-Signal über diese Schaltung an das Kontroll-Zählgerät gegeben wird. Die Zahl der registrierten Impulse muss dann der Gleichung

$$
Z=\Delta T \cdot f+1
$$

genügen, wenn $\Delta T$ die Intervallänge in Sekunden, $f$ die Frequenz des Mutteroszillators in 1/Sekunden und $Z$ die Zahl der registrierten Impulse bedeutet.
Durch Herausführen der Ausgangsimpulse der einzelnen ElT-Stufen beim $10 \mathrm{kHz}$-Betrieb an entsprechende Ausgangsklemmen über Anpassungskathodenfolger stehen Zeitsignale-je nach Wunsch 10000 , $1000,100,10$ und 1 Hertz-zu beliebiger Verwendung. Beispielsweise können mit dem Sekundensignal wesentlich vereinfachte Zeitgeber der im Absatz 3.1 beschriebenen Art versorgt werden und dadurch für viele Messstellen gleiche Zeitintervalle bzw. Zeitintervalle gleicher Ungenauigkeit abgegeben werden.

\subsection{Die Spannungsversorgung}

Das Blockschaltbild der Spannungsversorgung ist in Abb. 12 wiedergegeben.

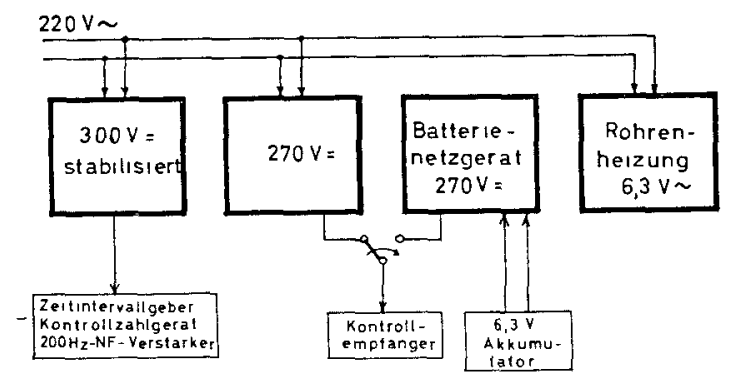

Abb. 12. Blockschaltbild Spannungsversorgung.

Das zur Versorgung des Kontrollempfängers bei erheblichem Störpegel des Lichtnetzes als Ausweichmöglichkeit vorgesehene Batterie-Netzgerät (Betriebsspannung: $6 \mathrm{~V}=$ ) besteht aus einem mit zwei $\mathrm{AD}$ 105/III bestücktem Transistor-Gegentaktoszillator der Frequenz $250 \mathrm{~Hz}$; die Niedervolt-Wechselspannung wird auf die Sollspannung hochtransformiert und in üblicher Weise gleichgerichtet.

Da ausser dem Kontrollempfänger (HF- und ZFTeil) alle Teile des Zeitgebers an einer Gleichspannungsversorgung liegen, mussten viele Schaltstufen durch ausreichende Siebschaltungen entkoppelt werden. Als besonders rückwirkungsempfindlich stellten sich die Startimpulskoinzidenzstufen sowie die elektronischen Schalter heraus.

\section{Fehlerbetrachtung}

Bei der Betrachtung der Messungenauigkeit des Zeitintervalles sind die vorausberechenbaren systematischen Fehler von den sonstigen streng zu unterscheiden.

\subsection{Systematische Fehler}

Bei der Durchführung der Kontrollmessung mit Hilfe des PTB-Normsignals ist zwar der Einschaltmoment durch Koinzidenz zwischen Start- und Signal- 
impuls bekannt (siehe Abb. 5), nicht aber die Phasenlage des letzten, vor dem Stopimpuls liegenden Signalimpulses. Der sich hieraus ergebende maximal mögliche Fehler beträgt eine Periodendauer des $200 \mathrm{~Hz}$-Signals, also $5 \mathrm{~ms}$. Weitere Fehler ergeben sich durch die endliche Breite des Koinzidenzsignals (ca $10 \mu \mathrm{s}$ ) und die Flankensteilheit des Elektronenschalters (ca $50 \mu \mathrm{s}$ ). Also ist der maximal mögliche Fehler der Kontrollmessung $5.06 \mathrm{~ms}$. Damit der relative Fehler klein wird, ergibt sich eine erforderliche Kontrollmesszeit, wenn der systematische relative Fehler gleich der Quarzungenauigkeit sein soll, von mindestens:

bei $\delta(\Delta T)=5 \mathrm{~ms}$ ist $T=\delta(\Delta T) 5 \times 10^{-5}=$ $=100$ Sekunden.

Für die Betrachtung des systematischen Fehlers des Zeitintervallgebers gelten die gleichen Überlegungen wie oben, nur dass wegen des jetzt zu betrachtenden $10 \mathrm{kHz}$-Steuersignals der maximal mögliche Fehler nur $160 \mu$ s beträgt. Da das beschriebene Gerät für Zeitintervalle im Minutenbereich vorgesehen ist, spielt dieser Fehler jedoch keine Rolle. Für spezielle Messvorhaben im Sekundenbereich wäre die Verwendung eines $100 \mathrm{kHz}$ - oder $1 \mathrm{MHz}-\mathrm{Mutteroszillators} \mathrm{ratsam}$.

Es sei noch einmal darauf hingewiesen. dass die eben beschriebenen Fehler nicht unbedingt auftreten müssen; nur entziehen sich die Abweichungen dieser Grössenordnungen der Kontrolle, falls sie auftreten. Die tatsächliche Ungenauigkeit des Zeitgebers wurde deshalb aus der statistischen Streuung vieler einzelner Messungen ermittelt.

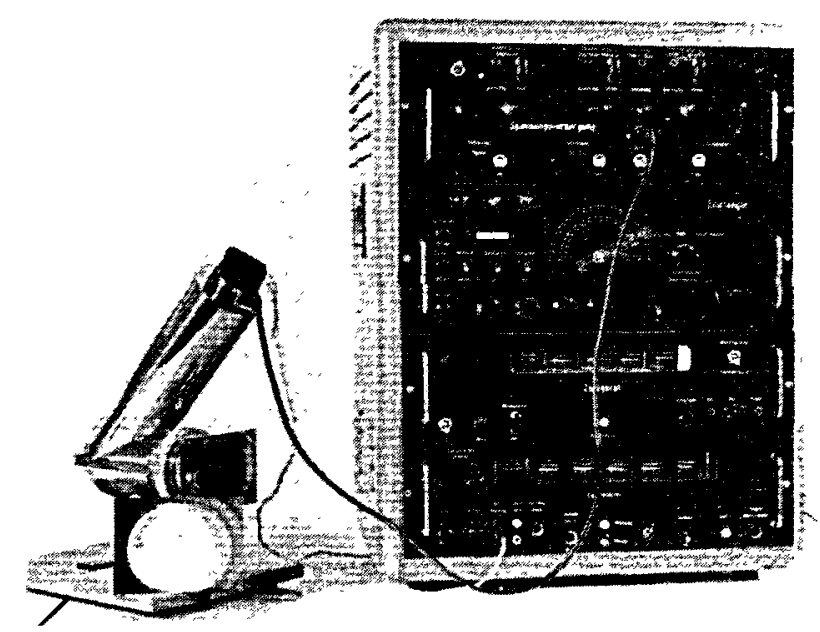

Abb. 13. Gesamtansicht des universellen Zeitgebers mit Stoppuhr-Eichvorrichtung.

\subsection{Gemessene Fehler}

Durch 100, jeweils zwei Minuten (eingestellt am Zeitgeber) dauernde Vergleichsmessungen mit dem PTB-Normsignal wurden folgende Ergebnisse gemessen: Mittelwert der innerhalb der Messzeit gespeicherten Normimpulse: 24001 (Sollwert: 24001); relative quadratische Abweichung vom Mittelwert, gemittelt über alle hundert Messungen: $6 \times 10^{-5}$. Dieser Fehler entspricht praktisch der Frequenzgenauigkeit des den Mutteroszillator steuernden Quarzes von $5 \times 10^{-5}$.

Herrn Dr. Hogrebe danke ich für sein Interesse an dieser Arbeit. Herrn H. Oppermann bin ich für die Durchentwicklung der Schaltungen dankbar. 